\title{
Impact of Receive Diversity on the Performance of Amplify-and-Forward Relaying under APS and IPS Power Constraints
}

\author{
Hakam Mheidat, Student Member, IEEE, and Murat Uysal, Member, IEEE
}

\begin{abstract}
In this letter, we investigate the impact of receive diversity on the error rate performance of a relay-assisted cooperative scheme in which the relay terminal is operating in amplify-and-forward (AF) mode under the so-called average power scaling (APS) and instantaneous power scaling (IPS) constraints. We assume that the source and relay terminals are each equipped with one antenna, while the destination terminal is equipped with $N$ receive antennas. Through the derivation of symbol error rate expressions, we demonstrate that the maximum achievable diversity orders under APS and IPS constraints are $N+1$ and $2 N$, respectively.
\end{abstract}

Index Terms-Cooperative diversity, amplify-and-forward relaying, error rate performance analysis.

\section{INTRODUCTION}

$\mathbf{C}$ OOPERATIVE diversity is an effective tool to exploit distributed spatial diversity in the wireless networks. The application of conventional space-time codes for user cooperation in the form of virtual antennas has recently drawn much attention, see [1], [2], [3] and the references therein. While most of these works build upon the assumption that each node is equipped with a single antenna, there have been recent information theoretical results which demonstrate the benefits of multiple antenna deployment [4]. Error rate performance analysis of distributed space-time codes with multiple-antenna nodes are presented in [5], [6] assuming decode-and-forward (DF) relaying. In this letter, we consider an AF relaying scenario where two single-antenna user nodes are communicating with a destination node equipped with $N$ receive antennas and investigate the effect of multiple-antenna deployment at the destination terminal upon the overall performance. For AF relaying, we assume two different power constraints [7], namely $\beta_{1}^{2}=1 / \underset{n, h_{S R}}{E}\left[\left|r_{R}\right|^{2}\right]$ and $\beta_{2}^{2}=1 / \underset{n}{E}\left[\left|r_{R}\right|^{2}\right]$, where $r_{R}$ is the received signal at the relay terminal and $E$ [.] denotes the expectation operation. In the first constraint, the expectation is with respect to both $n$ (which models the additive noise term) and $h_{S R}$ (which models the fading coefficient in the sourceto-relay link). This ensures that an average output power is maintained, but allows for the instantaneous output power to

Manuscript received December 17, 2005. The associate editor coordinating the review of this letter and approving it for publication was Prof. George Karagiannidis. This work was supported in part by an NSERC Special Opportunity Grant (SROPJ305821-05).

The authors are with the Dept. of Electrical and Computer Engineering, University of Waterloo, Ontario, Canada, N2G3L1 (e-mail: \{hmheidat, muysal\}@ece.uwaterloo.ca.

Digital Object Identifier 10.1109/LCOMM.2006.06028.

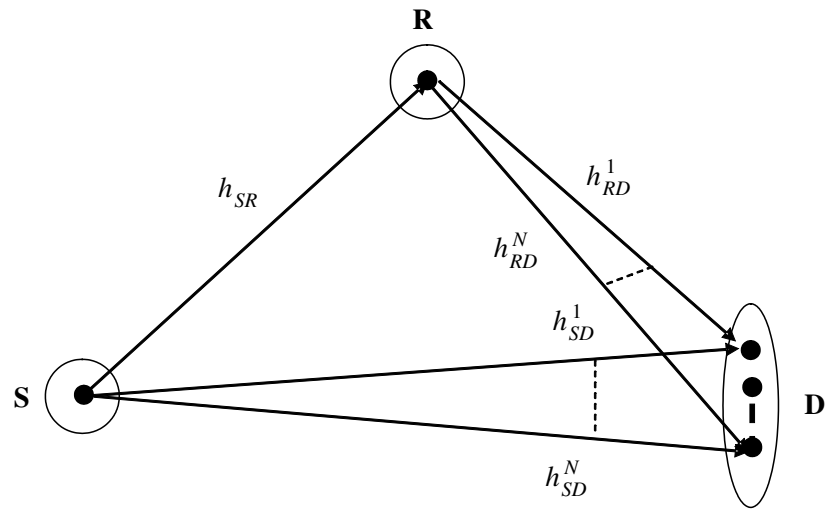

Fig. 1. Schematic representation of relay-assisted transmission with $N$ receive antennas at the destination terminal.

be much larger than the average. In the second constraint, the expectation is carried only over $n$ while each realization of $h_{S R}$ needs to be estimated and utilized in the computation of scaling term. This ensures that the same output power is maintained for each realization. We refer the first and second constraints as average power scaling (APS) and instantaneous power scaling (IPS) constraints, respectively. In this letter, we derive symbol error rate (SER) expressions for AF relaying with receive diversity (i.e. destination terminal with multiple antennas) under these two power constraints and demonstrate the maximum achievable diversity orders.

\section{TRANSMISSION MODEL}

We consider the relay-assisted transmission scenario in Fig. 1. The source terminal communicates with the relay and destination terminals during the first signaling interval. In the second signaling interval, only the relay communicates with the destination terminal. This set-up is named as Protocol II in [1] and originally proposed in [7]. Let the M-PSK modulation signal transmitted by the source terminal during the first time slot denoted as $x$. The signal received at the relay terminal is given as

$$
r_{R, 1}=\sqrt{E_{S R}} h_{S R} x+n_{R, 1}
$$

In the first time slot, the signal received at the $i^{t h}$ receive antenna of the destination terminal is

$$
r_{D, 1}^{i}=\sqrt{E_{S D}} h_{S D}^{i} x+n_{D, 1}^{i}, i=1,2, \ldots, N
$$


In the second time slot, the relay terminal transmits a scaled version of its received signal $r_{R, 1}$. The received signal at the $i^{\text {th }}$ receive antenna is, therefore, given by

$$
r_{D, 2}^{i}=\sqrt{E_{R D}} h_{R D}^{i} \beta_{j} r_{R, 1}+n_{D, 2}^{i}, i=1,2, \ldots, N
$$

where the scaling term $\beta_{j}, j=1,2$, is

$$
\beta_{j}^{2}=\left\{\begin{array}{l}
1 /\left(E_{S R}+N_{0}\right), \text { for APS } \\
1 /\left(E_{S R}\left|h_{S R}\right|^{2}+N_{0}\right) \text { for IPS }
\end{array}\right.
$$

In the above, $n_{R, 1}, n_{D, 1}^{i}$, and $n_{D, 2}^{i}$ are the independent samples of a zero-mean complex Gaussian random variable with variance $N_{0} / 2$ per dimension, which model the additive noise term. $E_{R D}, E_{S D}$, and $E_{S R}$ represent the average energies available at the destination and relay terminals, taking into account for possibly different path loss and shadowing effects in relay-to-destination $(R \rightarrow D)$, source-to-destination $(S \rightarrow D)$ and source-to-relay $(S \rightarrow R)$ links. $h_{R D}^{i}, h_{S D}^{i}$, and $h_{S R}^{i}$ denote the complex fading coefficients over $R \rightarrow D$, $S \rightarrow D$, and $S \rightarrow R$ links, respectively. They are modeled as independent and identically distributed (i.i.d) complex Gaussian random variables with variance 0.5 per dimension leading to the well-known Rayleigh channel assumption. Under APS power constraint assumption, we can rewrite (3) after normalization as in [1]

$$
r_{D, 2}^{i}=\sqrt{\gamma^{i}} \sqrt{E_{R D}} h_{S R} h_{R D}^{i} x+n
$$

where $n$ is a zero-mean complex Gaussian random variable with variance $N_{0} / 2$ per dimension and $\gamma^{i}$ is defined as

$$
\gamma^{i}=\frac{E_{S R} / N_{0}}{1+E_{S R} / N_{0}+\left|h_{R D}^{i}\right|^{2} E_{R D} / N_{0}}
$$

Under IPS constraint, the received signal preserves the same form as in (4) where $\gamma^{i}$ is now given as

$$
\gamma^{i}=\frac{E_{S R} / N_{0}}{1+\left|h_{S R}\right|^{2} E_{S R} / N_{0}+\left|h_{R D}^{i}\right|^{2} E_{R D} / N_{0}}
$$

\section{DeRIVATION OF SER EXPRESSIONS}

The destination terminal collects the received signals over the first and second time slots with a maximum ratio combiner (MRC). For fixed channel realizations, the instantaneous SNR at the MRC output is $\gamma_{T}=\gamma_{S \rightarrow D}+$ $\gamma_{S \rightarrow R \rightarrow D}$, where $\gamma_{S \rightarrow D}=\left(E_{S D} / N_{0}\right) \sum_{i=1}^{N}\left|h_{S D}^{i}\right|^{2}$ and $\gamma_{S \rightarrow R \rightarrow D}=\left(E_{R D} / N_{0}\right) \sum_{i=1}^{N} \gamma^{i}\left|h_{S R}\right|^{2}\left|h_{R D}^{i}\right|^{2}$ represent the instantaneous SNRs in $S \rightarrow D$ and $S \rightarrow R \rightarrow D$ links, respectively. In the following, we present SER derivations under APS and IPS constraints:

\section{A. SER under APS constraint}

Due to the presence of $\left|h_{R D}^{i}\right|^{2}$ term in (5), the derivation of SER becomes analytically difficult unless some assumptions are imposed on the SNR in the underlying links. In the following, we assume perfect power control where $S \rightarrow D$ and $R \rightarrow D$, links are balanced, $E_{R D} / N_{0}=E_{S D} / N_{0}$, and sufficiently large SNR for the $S \rightarrow R$ link, i.e. $E_{S R} / N_{0}>$
$E_{S D} / N_{0}$. Under these assumptions, we have $\gamma^{i} \approx 1$ and $\gamma_{S \rightarrow R \rightarrow D}=\left(E_{R D} / N_{0}\right)\left|h_{S R}\right|^{2} \sum_{i=1}^{N}\left|h_{R D}^{i}\right|^{2}$. Relying on previous results reported in [8] for the SER performance of coherent M-PSK, we can write

$$
P=\left.\frac{1}{\pi} \int_{0}^{(M-1) \pi / M} \Phi_{\gamma_{T}}(-s)\right|_{s=\frac{\sin ^{2}(\pi / M)}{\sin ^{2} \theta}} d \theta
$$

where $\Phi_{\gamma_{T}}(-s)=\Phi_{\gamma_{S \rightarrow D}}(-s) \Phi_{\gamma_{S \rightarrow R \rightarrow D}}(-s)$ is the moment generating function (MGF) of $\gamma_{T}$. The MGF of $\gamma_{S \rightarrow D}$ can be readily found as $\Phi_{\gamma_{S \rightarrow D}}(-s)=\left(1+s E_{S D} / N_{0}\right)^{-N}$ [8]. The MGF of $\gamma_{S \rightarrow R \rightarrow D}$ can be evaluated as [9]

$$
\Phi_{\gamma_{S \rightarrow R \rightarrow D}}(s)=\int_{0}^{\infty} f_{Z_{1}}\left(z_{1}\right) \Phi_{Z_{2}}\left(s z_{1}\right) d z_{1}
$$

where we define $\gamma_{S \rightarrow R \rightarrow D}=Z_{1} Z_{2}$ in terms of $Z_{1}=$ $\sum_{i=1}^{N}\left|h_{R D}^{i}\right|^{2}$ and $Z_{2}=\left|h_{S R}\right|^{2} E_{S D} / N_{0}$. Here, $Z_{1}$ is a chisquared random variable with $2 N$ degrees of freedom [10], and $Z_{2}$ has the MGF $\Phi_{Z_{2}}\left(s z_{1}\right)=1 /\left(1-z_{1} s E_{S D} / N_{0}\right)$ [8]. This leads to

$$
\Phi_{\gamma_{S \rightarrow R \rightarrow D}}(-s)=\frac{1}{\Gamma(N)} \int_{0}^{\infty} \frac{z_{1}^{N-1} e^{-z_{1}}}{1+z_{1} s E_{S D} / N_{0}} d z_{1}
$$

where $\Gamma(\cdot)$ denotes the gamma function [11]. Using the results in $[11$, p.338, 3.353.5] to solve the resulting integral, we obtain the final SER expression in the form of a single finite-range integral as

$$
\begin{aligned}
& P=\frac{1}{\pi} \int_{0}^{(M-1) \pi / M} \frac{-1}{\Gamma(N)} \\
& \times\left[\frac{\left((-1)^{N-2} \xi^{N-1} e^{\xi} \Gamma(0, \xi)-\sum_{j=1}^{N-1}(j-1) !(-\xi)^{N-1-j}\right)}{\left(1+\frac{1}{\xi}\right)^{N} \frac{1}{\xi}}\right] d \theta
\end{aligned}
$$

where $\Gamma(\cdot, \cdot)$ denotes the incomplete gamma function [11] and $\xi=\sin ^{2} \theta\left(\sin ^{2}(\pi / M) E_{S D} / N_{0}\right)^{-1}$.

To have further insight into the SER performance, we upper bound (9) by replacing $\theta$ with $\pi / 2$ to yield Chernoff bound as

$$
P \leq \frac{K(M-1)}{M} \frac{1}{\left(1+E_{S D} / 2 N_{0}\right)^{N}} \frac{1}{E_{S D} / 2 N_{0}}
$$

where $K$ is defined as in (11). For asymptotically high $E_{S D} / N_{0}$, we obtain

$$
P \leq\left\{\begin{array}{cc}
\frac{M-1}{M} \Gamma\left(0, \frac{1}{E_{S D} / 2 N_{0}}\right)\left(\frac{E_{S D}}{2 N_{0}}\right)^{-2}, & N=1 \\
\frac{M-1}{M} \frac{\Gamma(N-1)}{\Gamma(N)}\left(\frac{E_{S D}}{2 N_{0}}\right)^{-(N+1)}, & N>1
\end{array}\right.
$$

Evidently, (12) reveals that the diversity order for AF relaying under APS constraint is $N+1$ and that full diversity (i.e. $2 N$ ) can not be exploited. This demonstrates that the smaller of the diversity orders experienced in $S \rightarrow R$ and $R \rightarrow D$ links becomes the performance bottleneck for the relaying path under this constraint. 


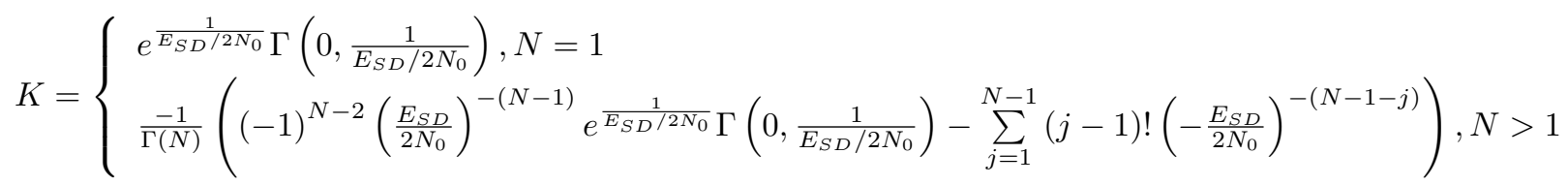

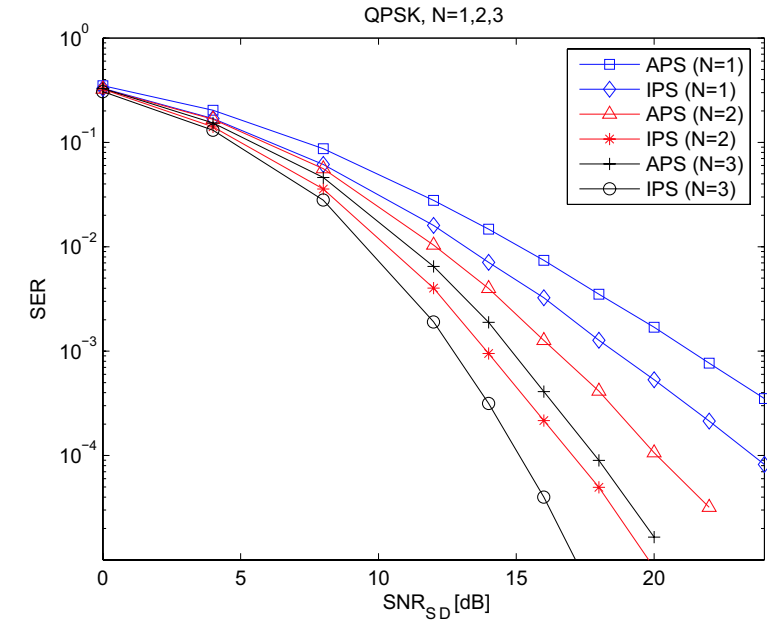

Fig. 2. SER performance of relay-assisted transmission with 1,2 , and 3 receive antennas.

\section{B. SER under IPS constraint}

Under the similar SNR assumptions imposed in the previous section, (6) reduces to $\gamma^{i} \approx 1 /\left|h_{S R}\right|^{2}$ which results in $\gamma_{S \rightarrow R \rightarrow D}=\left(E_{R D} / N_{0}\right) \sum_{i=1}^{N}\left|h_{R D}^{i}\right|^{2}$. Following similar steps to above, we can obtain the final SER expression as

$$
P=\frac{1}{\pi} \int_{0}^{(M-1) \pi / M}\left(1+\frac{\sin ^{2}(\pi / M)}{\sin ^{2} \theta} \frac{E_{S D}}{N_{0}}\right)^{-2 N} d \theta
$$

Replacing $\theta$ by $\pi / 2$ and assuming high $E_{S D} / N_{0}>>1$, we obtain an upper bound on SER as

$$
P \leq \frac{M-1}{M}\left(\frac{E_{S D}}{2 N_{0}}\right)^{-2 N}
$$

which demonstrates that the diversity order of AF relaying under IPS constraint is $2 N$. This is also equal to the maximum diversity available for the considered scenario.

For $N=1$ (i.e. destination terminal with single-antenna), diversity orders under both APS and IPS constraints become equal to 2. Although, asymptotically, full diversity is achieved for $N=1$ under both constraints, it can be further argued that AF-APS suffers from a coding gain loss (i.e. horizontal shift in the performance) and is outperformed by AF-IPS noting the additional term in (12) compared to (14), i.e. $\Gamma\left(0,1 /\left(E_{S D} / 2 N_{0}\right)\right)>1$.

\section{Simulation Results}

In this section, we present Monte-Carlo simulation results to confirm our analytical observations. We consider 4-PSK modulation and assume that $E_{S D} / N_{0}$ is scaled by the number of receive antennas, i.e. by $N$. We also assume $E_{S D}=E_{R D}$, i.e. $S \rightarrow D$ and $R \rightarrow D$ links are balanced. This can be achieved through power control. As for the $S \rightarrow R$ link, we assume $E_{S R} / N_{0}=35 \mathrm{~dB}$. Fig. 2 illustrates the simulated SER performance for $N=1,2,3$ receive antennas. For all considered cases, derived SER expressions, i.e. (9) and (13) perfectly match with the simulation results and are omitted here for the sake of illustration. It is observed from Fig. 2 that the diversity orders for the single-antenna case under both APS and IPS constraints are equal to 2. AF-IPS still outperforms AF-APS confirming our earlier analytical observations. For $N=2$ and 3, AF-IPS achieves diversity orders of 4 and 6 , exploiting the available full diversity (i.e. $2 N$ ) in the underlying channels as predicted by (14). On the other hand, AF-APS is able to exploit only a partial diversity, achieving diversity orders of 3 and 4 for $N=2$ and 3 , respectively. This partial diversity is equal to $N+1$ as predicted by (12).

\section{Conclusions}

We have derived SER expressions for AF relaying under the so-called APS and IPS constraints and investigated the impact of receive diversity on the error rate performance. Our analysis reveals out that the diversity order of AF-APS over the relaying path is governed by the link which has smaller diversity order. This results in an overall diversity order of $N+1$ for the considered cooperative scenario. For the same scenario, AF-IPS is able to exploit the full available diversity and, therefore, outperforms AF-APS.

\section{REFERENCES}

[1] R. U. Nabar, H. Boelcskei, and F. W. Kneubhueler, "Fading relay channels: performance limits and space-time signal design," IEEE J. Select. Areas Commun., vol. 22, pp. 1099-1109, Aug. 2004.

[2] T. A. Tsiftsis, G. K. Karagiannidis, S. A. Kotsopoulos, and F.-N. Pavlidou, "BER analysis of collaborative dual-hop wireless transmissions," IEE Electron. Lett., vol. 40, pp. 1732-1745, May 2004.

[3] G. K. Karagiannidis, T. A. Tsiftsis, and R. K. Mallik, "Bounds for multi-hop relayed communications in Nakagami-m fading," IEEE Trans. Commun., vol. 54, pp. 18-22, Jan. 2006.

[4] B. Wang, J. Zhang, and A. Host-Madsen, "On the capacity of MIMO relay channels," IEEE Trans. Inform. Theory, vol. 51, p. 29-43, Jan. 2005.

[5] A. Stefanov and E. Erkip, "On the performance analysis of cooperative space-time coded systems," in Proc. IEEE WCNC 2003, pp. 729-734.

[6] S. Yiu, R. Schober, and L. Lampe, "Distributed space-time block coding for cooperative networks with multiple-antenna nodes," in Proc. IEEE Workshop on CAMSAP 2005, pp. 52-55.

[7] J. N. Laneman, "Cooperative diversity in wireless networks: algorithms and architectures," Ph.D. dissertation, Massachusetts Institute of Technology, Cambridge, MA, Aug. 2002.

[8] M. K. Simon and M.-S. Alouini, Digital Communication over Fading Channels: A Unified Approach to Performance Analysis. New York: John Wiley \& Sons, 2000.

[9] A. Stuart and J. K. Ord, Kendall's Advanced Theory of Statistics, 5th ed. London: Griffin and Co., 1987.

[10] J. G. Proakis, Digital Communications, 2nd ed. New York: McGrawHill, 1989.

[11] I. S. Gradshteyn and I. M. Ryzhik, Table of Integrals, Series and Products, 5th ed. London: Academic Press, 1980. 